\title{
E3 Ubiquitin-Protein Ligase ARIH1
}

National Cancer Institute

\section{Source}

National Cancer Institute. E3 Ubiquitin-Protein Ligase ARIH1. NCI Thesaurus. Code C134189.

E3 ubiquitin-protein ligase ARIH1 (557 aa, 64 kDa) is encoded by the human ARIH1 gene. This protein is involved in protein ubiquitination. 